\title{
Altered DNA methylation in neonates born large-for-gestational- age is associated with cardiometabolic risk in children
}

\author{
Xian-hua Lin ${ }^{1,2, *}$, Dan-dan Wu ${ }^{1,2, *}$, Ling Gao ${ }^{1,2, *}$, Jun-yu Zhang ${ }^{1,2}$, Hai-tao Pan ${ }^{3}$, \\ Hui Wang ${ }^{5}$, Cheng Li ${ }^{1,2}$, Ping Zhang ${ }^{1}$, Meng-xi Guo ${ }^{3}$, Yan-ting Wu ${ }^{1,2}$, Ya-jing Tan ${ }^{1,2}$, \\ Li Jin ${ }^{1,2}$, Yu-qian Xiang ${ }^{1,2}$, Ju-Xue Li ${ }^{1,2}$, Jian-zhong Sheng ${ }^{3,4}$, He-feng Huang ${ }^{1,2,3}$ \\ ${ }^{1}$ The International Peace Maternity and Child Health Hospital, School of Medicine, Shanghai Jiao Tong University, Shanghai, \\ China \\ ${ }^{2}$ Institute of Embryo-Fetal Original Adult Disease, Affiliated to Shanghai Jiao Tong University, School of Medicine, Shanghai, China \\ ${ }^{3}$ The Key Laboratory of Reproductive Genetics, Ministry of Education, Zhejiang University, Hangzhou, China \\ ${ }^{4}$ Department of Pathology and Pathophysiology, School of Medicine, Zhejiang University, Hangzhou, China \\ ${ }^{5}$ Department of Obstetrics and Gynecology, Meihua Central Hospital, Shanghai, China \\ *These authors contributed equally to this work
}

Correspondence to: He-feng Huang, email: huanghefg@sjtu.edu.cn

Keywords: neonate, large-for-gestational-age, DNA methylation, preschool children, cardiometabolic risk

Received: July 26, 2016

Accepted: November 07, 2016

Published: November 18, 2016

\section{ABSTRACT}

Background: Infants being born Large-for-gestational-age (LGA) are prone to developing cardiometabolic disease. However, the underlying mechanisms remain unclear.

Results: Clinical investigation showed that children born LGA had significantly higher serum level of total cholesterol (TC), low-density lipoproteincholesterol (LDL-C), and insulin, ratio of TC/high-density lipoprotein-cholesterol (HDL-C) compared to children born appropriate for gestational age (AGA). Birth weight (BW) was positively correlated to TC, LDL-C, and the ratio of TC/ HDL in serum. Genome-wide DNA methylation analyzed in umbilical cord blood of controls and macrosomia cases. We identified 3459 methylation variable positions (MVPs) achieving genome-wide significance (adjusted $P$-value $<0.05$ ) with methylation differences of $\geq 5 \%$. A total of 327 MVPs were filtered by methylation differences of $\geq 7 \%$ located within an island, which mapped to 213 genes. Function analysis using Ingenuity Pathway Analysis showed 16 genes enriched in "cardiovascular disease". Four genes included contributed to hyperlipidemia.

Materials And Methods: Fifty-eight children aged 3-6 years born LGA and 123 subjects born AGA were enrolled. Anthropometric parameters and blood pressure (BP) were measured, and metabolic assessment was performed in all subjects. Genomewide DNA methylation in umbilical blood was assayed by the $450 \mathrm{~K}$ BeadChip in six AGA and six macrosomia newborns.

Conclusions: Our data indicate that excess birth weight may increase the risk of lipid dysfunction in children aged 3-6 years. It might through reprogramming a group of genes correlated to cardiovascular disease. The genes identified in this study might be potential biomarker for cardiometabolic disease.

\section{INTRODUCTION}

Prenatal and early postnatal origins of cardiometabolic disease have been extensive studied in the past a few years. The gestational stage and early childhood represents a window of phenotypic plasticity and is a sensitive period related to programming cardiometabolic risk. Women with increased body mass index (BMI) and obesity during pregnancy commonly result in fetal overgrowth and the birth of a large-for-gestational age 
(LGA) infant [1]. Infants being born LGA are prone to developing obesity, diabetes, and hypertension during childhood and later in life $[2,3]$. Being born LGA to mothers with or without gestational diabetes mellitus (GDM) and obesity is associated with diverse effects on cardiometabolic risk factors at prepuberty [4-6]. Other studies have shown that high birth weight and rapid weight gain in childhood are associated with cardiometabolic risk later in life [7-10].

Cumulative evidences suggest that there is a relationship between excess birth weight and metabolic syndrome (MetS) and cardiovascular disease (CVD) risk factors $[4,6]$. However, the underlying mechanisms have not yet been clearly demonstrated. MetS and CVD have a heritable component that is not attributable to genetic factors. Instead, epigenetic mechanisms may have an additional role in mediating inheritance of disease risk [11]. Prenatal exposure to famine during the Dutch hunger winter of 1944 is associated with obesity with less DNA methylation of the imprinted insulin-like growth factor 2 (IGF2) gene in exposed offspring relative to their unexposed siblings $[12,13]$. Recently, retinoid X receptor alpha $(R X R A)$ promoter methylation was demonstrated to correlate with increased adiposity in two independent cohorts of children of mothers with low carbohydrate intake [14]. Increasing evidence suggests that epigenetic marks can be subjected to reprogramming in response to both stochastic and environmental stimuli, such as the in utero environment [15]. In addition, a large number of epigenetic marks are relatively stable over time [16], suggesting that those epigenetic changes acquired early in life may have a long-lasting impact on future health.

The objective of the present study was to assess the association between altered DNA methylation in neonates born with high birth weight and cardiometabolic risk parameters in preschool children, using a casecontrol study of preschool children born at term after noncomplicated pregnancies.

\section{RESULTS}

\section{Metabolic parameters}

To compare the metabolic parameters between children born with different birth weight, we measured glucose and insulin values, HOMA index, lipid profile, and uric acid in fasting conditions in children at 3-6 years of age. As shown in Table 1, when grouped by birth weight categories, the LGA children had significantly higher levels of total cholesterol (TC), low-density lipoprotein cholesterol (LDL-c), and insulin and ratio of TC/high-density lipoprotein cholesterol (HDL-c) when compared with that for the appropriate for gestational age (AGA) children; their body weight, length, and BMI were also higher. Although there was a trend to higher levels of glucose, it was not significant. Furthermore, maternal characteristics of those subjects are presented in Supplementary Table S1. Due to the matching criteria, maternal age, height, weight gain during pregnancy, and maternal occupation, education, parity, and family history showed no significance between both groups. Furthermore, there was no difference in blood pressure (BP), red blood cells, hemoglobin, platelets and serum glucose, and protein levels between mothers of AGA and LGA children. However, the weight and BMI during late pregnancy of mothers from LGA group presented significantly higher than that of mothers from the AGA group. Maternal triglyceride (TG) levels were higher in the LGA group than that in AGA group (Supplementary Table S1), whereas there was no difference in cholesterol levels.

\section{Relationship between anthropometric, BP, and metabolic parameters at 3-6 years of age}

To further study the relationship between the birth weight and cardiometabolic risks, we analyzed the correlation coefficients between birth and current weight, as well as the mean weight gain, with systolic and diastolic BP and cardiometabolic parameters. Birth weight was related to cardiometabolic parameters, positively to TC, LDL-c, and the ratio of TC/HDL-c even after being adjusted by BMI and weight at 3-6 years of age (Table 2). The weight and BMI at 3-6 years old and the average weight gain were positively related to systolic and diastolic BP, fasting glucose, insulin, and HOMA index but not correlated with TC, LDL-c, and the ratio of TC/HDL-c (Supplementary Table S2). Those results suggest that excess birth weight increases the risk of cardiometabolic disease later in life.

\section{DNA methylation analyses}

To explore the mechanisms involved in birth weight and the risk of cardiometabolic disease in later life, we use the Illumina Human Methylation 450 BeadChip to assay genome-wide DNA methylation in umbilical cord blood from six controls and six macrosomia cases. Detailed information for the 6 pairs of selected subjects was showed in Supplementary Table S3. Results indicated that there was comparable between the two groups. Totally, 444,152 $\mathrm{CpG}$ loci remained for analysis after all quality control steps. We set the threshold of significance for methylation variable positions (MVPs) using adjusted $P$ value $=0.05$ and delta beta $=5 \%$. Samples were successfully differentiated into control and macrosomia groups after principal component analysis. In total, there were 3459 MVPs remaining after the initial filter, and 62.3\% (2156/3459) of them were less methylated. According to their different genomic features and relation to the nearest CpG islands, MVPs could be annotated as transcription start sites (TSS) 1500, TSS200, 1stExon, 5'untranslated regions (UTR), 3'UTR, gene body or intergenic region 
Table 1: Anthropometric and metabolic parameters in children of 3-6 years grouped by birth weight

\begin{tabular}{|c|c|c|c|}
\hline & $\operatorname{AGA}(n=123)$ & LGA $(n=58)$ & $P$ value \\
\hline Age (month) & $55.11 \pm 7.87^{2}$ & $55.02 \pm 7.20$ & 0.472 \\
\hline Birth weight (g) & $3237.07 \pm 322.88$ & $4114.05 \pm 197.45$ & $<0.001$ \\
\hline Birth height $(\mathrm{cm})$ & $50.06 \pm 1.68$ & $50.90 \pm 1.55$ & $<0.001$ \\
\hline Male/female sex (n) & $64 / 59$ & $32 / 26$ & 0.751 \\
\hline Weight (kg) & $17.84 \pm 2.75$ & $19.75 \pm 3.07$ & $<0.001$ \\
\hline Height $(\mathrm{cm})$ & $107.59 \pm 6.41$ & $110.41 \pm 4.86$ & 0.002 \\
\hline $\operatorname{BMI}\left(\mathrm{kg} / \mathrm{m}^{2}\right)$ & $15.36 \pm 1.49$ & $16.14 \pm 1.76$ & 0.001 \\
\hline Weight gain (g/month) & $266.45 \pm 43.65$ & $287.36 \pm 62.46$ & 0.005 \\
\hline Systolic BP (mmHg) & $97.59 \pm 10.48$ & $98.94 \pm 6.96$ & 0.187 \\
\hline Diastolic BP (mmHg) & $56.92 \pm 8.55$ & $58.07 \pm 7.40$ & 0.190 \\
\hline MAP (mmHg) & $70.47 \pm 8.10$ & $71.69 \pm 6.19$ & 0.156 \\
\hline Pulse pressure (mmHg) & $40.67 \pm 9.41$ & $40.87 \pm 8.04$ & 0.446 \\
\hline Serum TG $(\mathrm{mmol} / \mathrm{L})$ & $0.73 \pm 0.28$ & $0.72 \pm 0.28$ & 0.487 \\
\hline Serum TC $(\mathrm{mmol} / \mathrm{L})$ & $4.31 \pm 0.74$ & $4.62 \pm 0.76$ & 0.005 \\
\hline Serum HDL-c (mmol/L) & $1.47 \pm 0.27$ & $1.47 \pm 0.24$ & 0.476 \\
\hline Serum LDL-c (mmol/L) & $2.25 \pm 0.54$ & $2.46 \pm 0.54$ & 0.008 \\
\hline Serum TC/HDL-c & $2.99 \pm 0.56$ & $3.19 \pm 0.57$ & 0.014 \\
\hline Fasting glucose $(\mathrm{mmol} / \mathrm{L})$ & $4.78 \pm 0.40$ & $4.87 \pm 0.37$ & 0.091 \\
\hline Fasting insulin (uU/ml) & $3.62 \pm 2.04$ & $4.19 \pm 1.99$ & 0.038 \\
\hline HOMA-IR & $0.79 \pm 0.50$ & $0.92 \pm 0.47$ & 0.048 \\
\hline
\end{tabular}

1. Data were analyzed by using Student's t, and Mann-Whitney tests.

2. Mean $\pm \mathrm{SD}$ (all such values).

3. BP, blood pressure.

4. MAP, mean arterial pressure.

(IGR), and island, shore, shelf, or open sea. Excluding $29.0 \%(1003 / 3459)$ MVPs that were located on an IGR, 23.8\% (585/2456), the remaining MVPs were distributed on islands (Figure 1A). Further analysis was performed in 327 MVPs (filtered by adjusted $p$ value $<0.05$, delta beta $\geq 7 \%$ ) and CpGs were located in islands, which mapped to 213 genes. Differential DNA methylation between controls and the macrosomia group could be observed in the heatmap (Figure 1B and 1C). DNA methylation level was down-regulated in $67.9 \%(222 / 327)$ of MVPs. To detect the biological functions and major pathways related to these 213 genes, we performed functional enrichment analysis using Gene Ontology (GO) Tool from DAVID Bioinformatics Resources. According to the results of GO enrichment in the biologic process (Supplementary Table S4), 4 of 5 terms were related to embryo organ or system development, and the top one was "embryonic organ development" ( 9 genes, $P=5.52 \mathrm{E}-04)$. "Endocytosis" ( 9 genes, $P=0.01$ ) was found to be the most significantly Kyoto Encyclopedia of Genes and Genomes (KEGG) term (Supplementary Table S5).

\section{Ingenuity pathway analysis (IPA) analysis}

We next used the IPA software (QIAGEN, Redwood City, CA, USA) for functional analyses for the 213 genes. We observed 27 enriched "Diseases and Disorders" terms (Figure 2). The top items identified were: (1) "Cancer", (2) "Organismal Injury and Abnormalities", and (3) "Endocrine System Disorders". In addition, "Cardiovascular Disease" (16 genes, $0.001<P<0.019$ ) was also included within the enrichment. Among 16 genes in "Cardiovascular Disease", many genes participated in lipid metabolism (Figure 3). Four representational genes, including Apolipoprotein $\mathrm{B}$ gene $(A P O B)$, Carboxylesterase 1 gene (CES1), Delta Like NonCanonical Notch Ligand 1 gene (DLK1), Lipase Maturation Factor 1, $(L M F 1)$, were contributed to hyperlipidemia. An average $10.1 \%$ lower methylation level was identified at 
Table 2: Correlation coefficients between cardiometabolic parameters with birth weight

\begin{tabular}{lcccc}
\hline \multirow{2}{*}{\multicolumn{1}{c}{ Parameters }} & \multicolumn{4}{c}{ Birth weight } \\
\cline { 2 - 5 } & Non-adjusted & $\boldsymbol{P}$ value & Adjusted & $\boldsymbol{P}$ value \\
\hline Systolic BP (mmHg) & 0.009 & 0.452 & 0.061 & 0.429 \\
Diastolic BP (mmHg) & -0.001 & 0.494 & -0.076 & 0.324 \\
MAP (mmHg) & 0.003 & 0.484 & 0.081 & 0.293 \\
Pulse pressure (mmHg) & 0.011 & 0.444 & 0.005 & 0.946 \\
Serum TG (mmol/L) & 0.054 & 0.237 & 0.025 & 0.749 \\
Serum TC (mmol/L) & $0.255^{*}$ & $<0.001$ & $0.267^{*}$ & $<0.001$ \\
Serum HDL (mmol/L) & -0.019 & 0.399 & -0.002 & 0.979 \\
Serum LDL (mmol/L) & $0.294^{*}$ & $<0.001$ & $0.302^{*}$ & $<0.001$ \\
Serum TC/HDL & $0.217^{\#}$ & 0.002 & $0.211^{\#}$ & 0.006 \\
Fasting glucose (mmol/L) & 0.022 & 0.383 & 0.043 & 0.575 \\
Fasting insulin (uU/ml) & 0.047 & 0.264 & 0.105 & 0.173 \\
HOMA-IR & 0.043 & 0.284 & 0.103 & 0.181 \\
\hline DBP
\end{tabular}

DBP indicates diastolic blood pressure; SBP, systolic blood pressure; MAP, mean arterial pressure; HDL, high-density lipoprotein; HOMA-IR, homeostatic model assessment; and LDL, low-density lipoprotein. Adjusted correlation coefficients, after adjusted by current weight and current BMI.

${ }^{*} P<0.001$, and ${ }^{\#} P<0.01$.

A
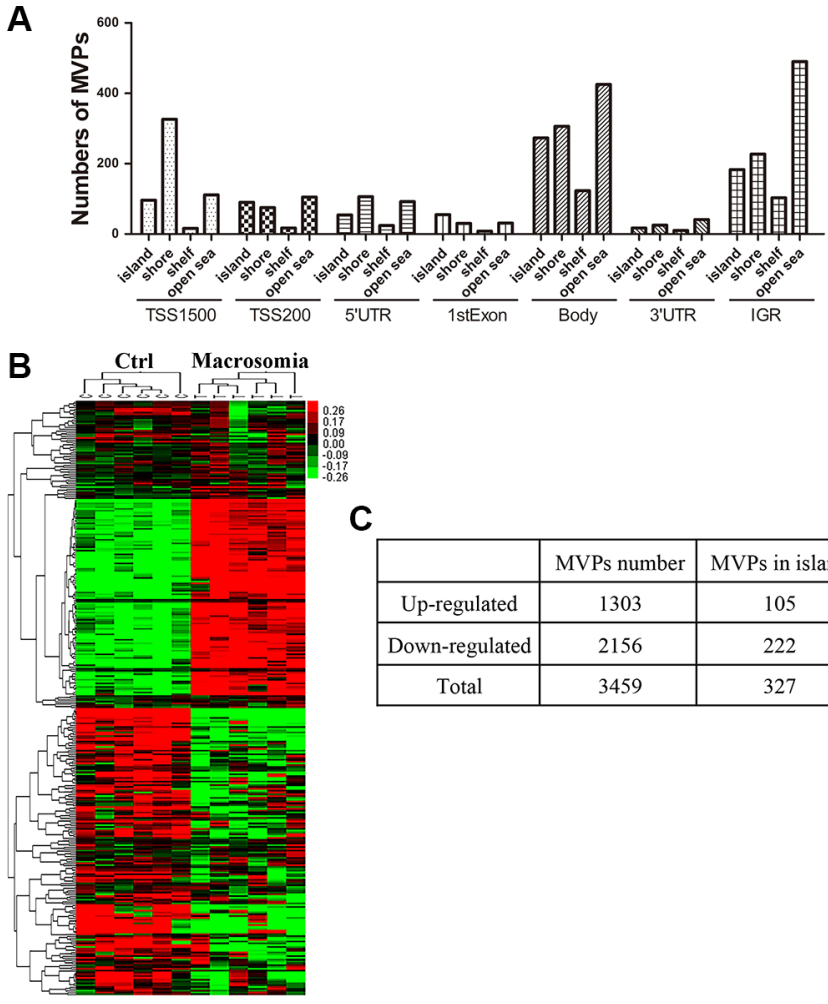

C

\begin{tabular}{|c|c|c|}
\hline & MVPs number & MVPs in island \\
\hline Up-regulated & 1303 & 105 \\
\hline Down-regulated & 2156 & 222 \\
\hline Total & 3459 & 327 \\
\hline
\end{tabular}

Figure 1: DNA methylation in genomic level altered in cord blood from macrosomia. (A) Distribution of a total of 3459 methylation variable positions (MVPs) after the initial statistical significant filter (adjusted $P$-value $<0.05$ and methylation differences of $\geq 5 \%$ ) according to epigenetic/genomic feature. $Y$-axis denotes specific numbers of MVPs involved in each epigenetic/genomic feature; $X$-axis denotes genomic features (annotated as TSS1500, TSS200, 1stExon, 5'UTR, 3'UTR, gene body or IGR, andepigenetic featuredistances from a CG enriched region (CGI) (island, shore, shelf, open sea). (Abbreviations: TSS1500, within $1.5 \mathrm{kB}$ of transcriptional start site; TSS200, within 200 bp of transcriptional start site; IGR, intergenic region). (B) Heat map including the top statistically significantly MVPs in island ( $n=327$, adjusted $P$-value $<0.05$ and methylation differences of $\geq 7 \%)$. (C) The number of up-regulated and downregulated MVPs with different filters. 
APOB DMR in Chr2: 21266944-21266969, version 2009 (GRCh37/hg19), which also was annotated asTSS200island; 21.2\% lower methylation level in Chr16: 55866890, at gene CES1 (body-island); 9.4\%, 10.1\%, and 12.9\% varied methylation level in Chr14: 101192852, 101192860, and 101192913, respectively, annotated as gene DLK1 (TSS1500-island); 15.8\%, 15.3\%, 20.2\%, and 23.0\% varied methylation level in Chr16: 979488, 979553, 979662, and 979898, respectively, annotated as gene $L M F 1$ (body-island). Arachidonate 15-Lipoxygenase gene ( $A L O X 15)$, along with $A P O B$, was referred to as fatty lesion associated in the "CVD" network, and $25.1 \%$ and $18.5 \%$ varied methylation level showed in Chr17: 4544507, 4544513 (body-island).

\section{Verification of the target genes}

Among those 16 genes in "CVD", we chose the first three genes according to the alphabetical order for verification with expanded samples (control: 22, macrosomia: 24). The methylation levels of five $\mathrm{CpG}$ sites of $A L O X 15,24 \mathrm{CpG}$ sites of $A P O B$, and $13 \mathrm{CpG}$ sites of CES1 were detected (Figure 4A-E). The average methylation level of ALOX15 in Chr17: 45445074544627 was significantly up-regulated in the macrosomia group (Figure 4B, $P=0.002$ ). $\mathrm{CpG}$ methylation levels at sites 1, 6-7 were significantly higher in the macrosomia group $(P<0.01)$, but the methylation levels at site 2,3 did not significantly differ $(P>0.05$ for all) (Figure 4A). No valid data were received at site 4 and 5 . The average methylation level of $A P O B$ in chr2: 21266623-21267021 in the macrosomia group was lower than that in the control group (Figure 4D, $P=0.02$ ). Differential $\mathrm{CpG}$ methylation levels lay at sites $1,15,16$, and $38(P<0.05)$ and sites 21 and 33-37 $(P<0.01)$, whereas the methylation levels of the rest of the 14 sites did not significantly differ $(P>0.05$ for all) (Figure 4C). No valid data were received at sites $2-7,22-28$, and 30 . The average methylation level of CES1 in chr16: 55866758-55867030 was significantly increased in the macrosomia group (Figure 4F, $P=0.0006)$. CpG methylation levels at sites $1-3,4,6,7$, $8,9-10,12,13$, and 14-15 were significantly higher in the macrosomia group $(P<0.01)$, but the methylation levels at site 14 and 15 did not significantly differ $(P>0.05)$ (Figure 4E). No valid data were received at site 5 and 11 .

\section{DISCUSSION}

Fetal origins of cardiovascular and metabolic diseases have attracted more and more attention. Cardiometabolic risk factors, including higher BP,

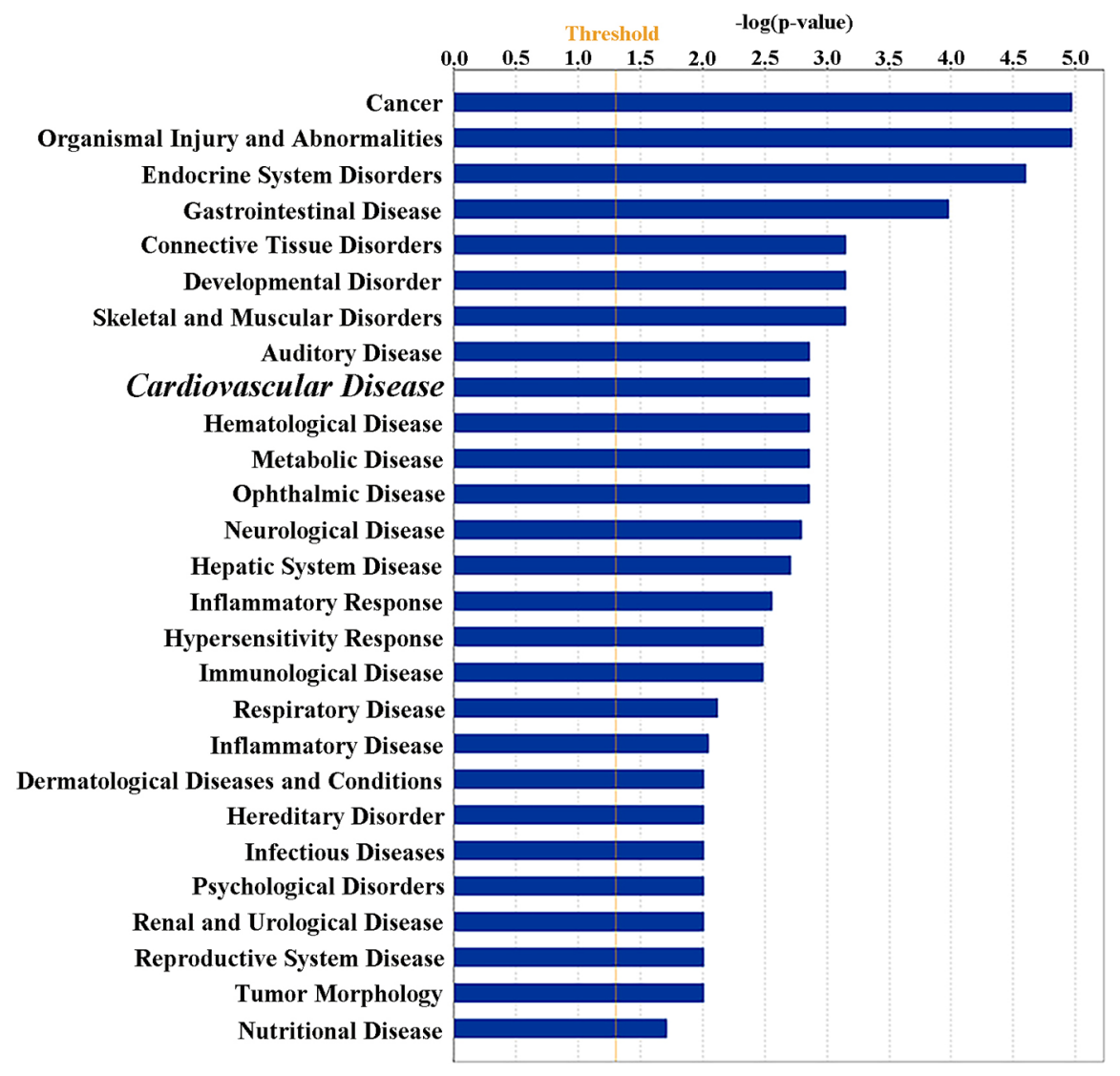

Figure 2: Ingenuity pathway analysis. Functional classification of 213 genes mapped by the top statistically significant 327 MVPs identified between controls and the macrosomia group using the Ingenuity Pathway Analysis. "Diseases and disorders" enriched 27 terms, and including "Cardiovascular Disease" (Bigger Italic fonts). 
triglycerides, TC and LDL-c, insulin, HDL-c, and ratio of TC to HDL-c are increasingly being determined in children as well as in adulthood [17-19]. The present prospective study performed in children born at term after a noncomplicated pregnancy shows that birth weight exerted independent influences on cardiometabolic parameters at preschool age.

Barker and his colleagues were the first to find significant associations between low birth weight and the risk of chronic diseases in adulthood, including coronary artery disease, hypertension and stroke, type 2 diabetes, and osteoporosis [20]. Strong evidence from several studies indicates that individuals born with a low birth weight are more likely to present cardiometabolic complications later in life [21-24]. Recent studies in the USA, Europe, and other countries have revealed a continuous increase of mean birth weight in the past 2 decades. So far, the long-term consequences of high birth weight have not been clearly defined. A recent review examined the role of high birth weight on the development of cardiometabolic consequences (obesity, body composition, type 2 diabetes mellitus, and CVD) in childhood and adulthood [25]. Overweight and overnutrition are among the most widely recognized risk factors of metabolic diseases. The findings in a systematic review suggest that an individual with high birth weight is prone to hypertension and higher BP during childhood [26]. The subjects included in this study were only 3-6 years old, we did not find any correlation between birth weight and BP. On the other hand, it is not surprise that the average weight gain and current weight were positively correlated to BP and insulin levels. The findings indicated that "catch-up" growth correlate with some aspects of a later MetS, such as BP, which implies that a catch-up growth may be another factor linked to hypertension later in life [27]. The fetal origins of the adult disease hypothesis propose that exposures to an adverse intrauterine environment directly relates to poor nutritional status in early life and may increase their risk of adult disease, such as metabolic and CVDs [28]. Here we showed the associations of high birth weight and altered DNA methylation in neonates with cardiometabolic risk parameters in preschool children. As we all know, there were complicated maternal risks linked to birth weight $[1,10,29]$. We also investigated the maternal information during pregnancy. The results showed in Supplementary Table S1 revealed that mothers with higher BMI and serum triglyceride levels during late pregnancy are apt to born LGA neonates, which imply that prenatal exposure to an adverse intrauterine environment
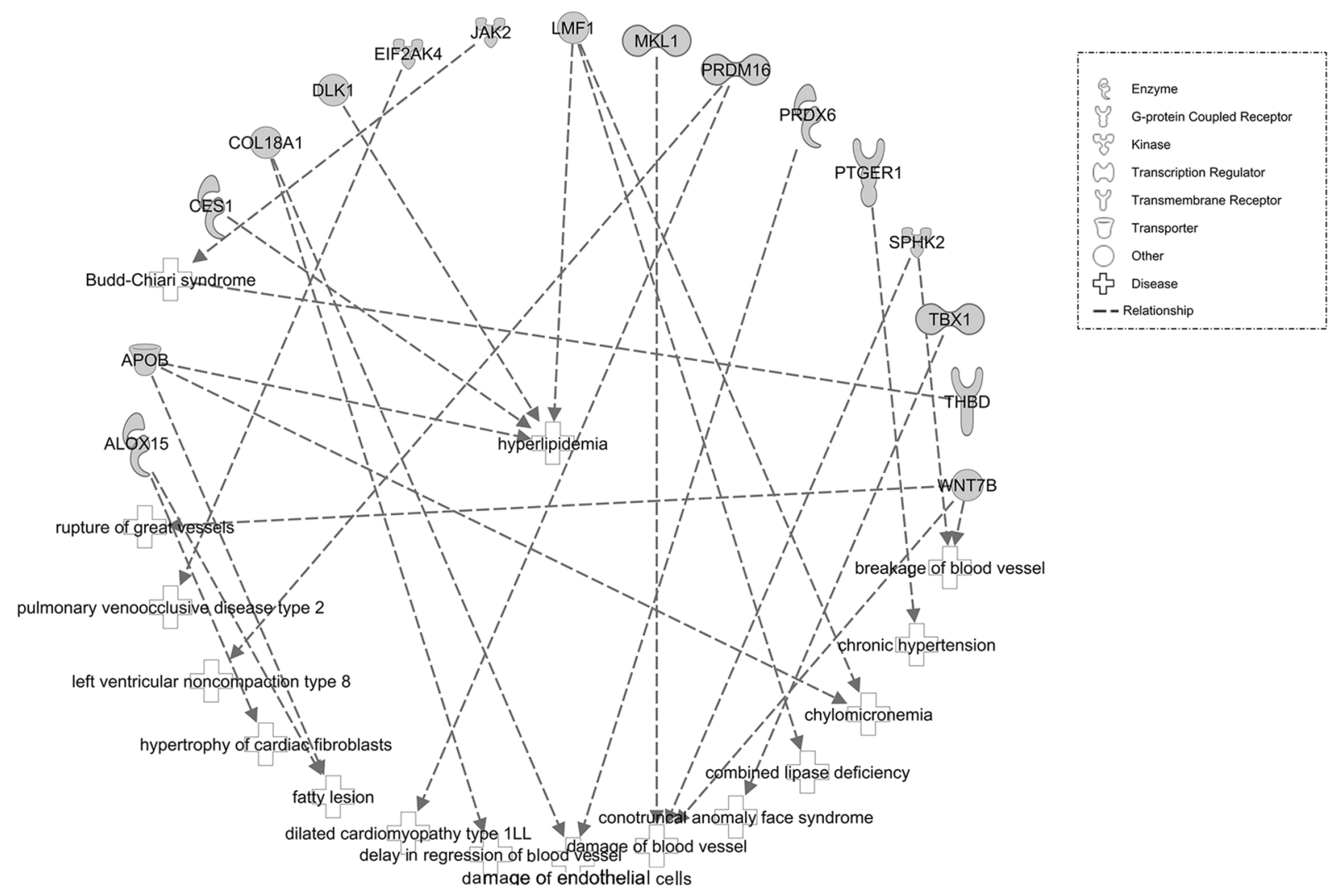

Figure 3: Downstream effect analysis of specific genes with differentially methylated CpGs associated with CVD. For this cardiovascular function network, genes or gene products are represented as nodes, and the biological relationship between two nodes is represented as an edge. All edges are supported by at least one publication in the Ingenuity Knowledge database. The legend of the interaction network and the relationships between molecules are summarized on the right of the figure. 
with potential consequences for subsequent developmental cardiometabolic diseases over the lifespan.

Accumulative epidemiological investigation has illuminated that macrosomia infants were more prone to develop CVD and MetS. However, the potential mechanisms were still unclear. Recently, epigenetics was suggested to provide a mechanistic link between environmental exposures and adult disease. DNA methylation is a well-known epigenetic modification that participates in metabolic programming during the perinatal period [30]. We have previously demonstrated in mice that the hyperglycemic intrauterine environment of GDM can increase the risk of diabetes in offspring by altering $\operatorname{Ig} f 2 / \mathrm{H} 19$ imprinting in islets [31]. Epigenetic marks can be subjected to reprogramming in response to in utero environment, which might lead to healthy or unhealthy phenotypes, thus enabling phenotypic plasticity in the context of a fixed genotype [15, 32]. Epigenetic variation in umbilical cord blood may have a mechanistic role in metabolic disease programming through interaction of the pregnancy environment with gene function $[29,33]$. Indeed, the results of DNA methylation analyses in umbilical cord blood in this study identified 327 MVPs with methylation differences of $>7 \%$ located within island, which mapped to 213 genes. Bioinformatics analysis showed many genes correlated to CVD and lipid metabolism.

The ChAMP package is a pipeline that integrates currently available $450 \mathrm{~K}$ analysis methods. ChAMPimplemented BMIQ was identified by Marabita as an effective method $[34,35]$. SNPs filter function available in this pipeline helps to prevent biases due to genetic variation in downstream statistical analyses aimed at identifying differentially methylated CpGs and focus investigation on the epigenetic factors. We also exclude probes on sex chromosomes to avoid their interference effect. However, the long period of clinical follow-up of women throughout pregnancy and their offspring in childhood was needed; it

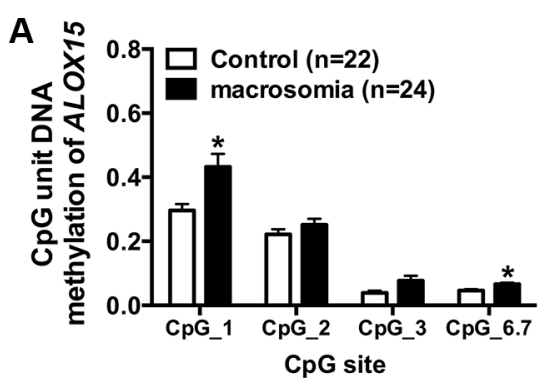

C
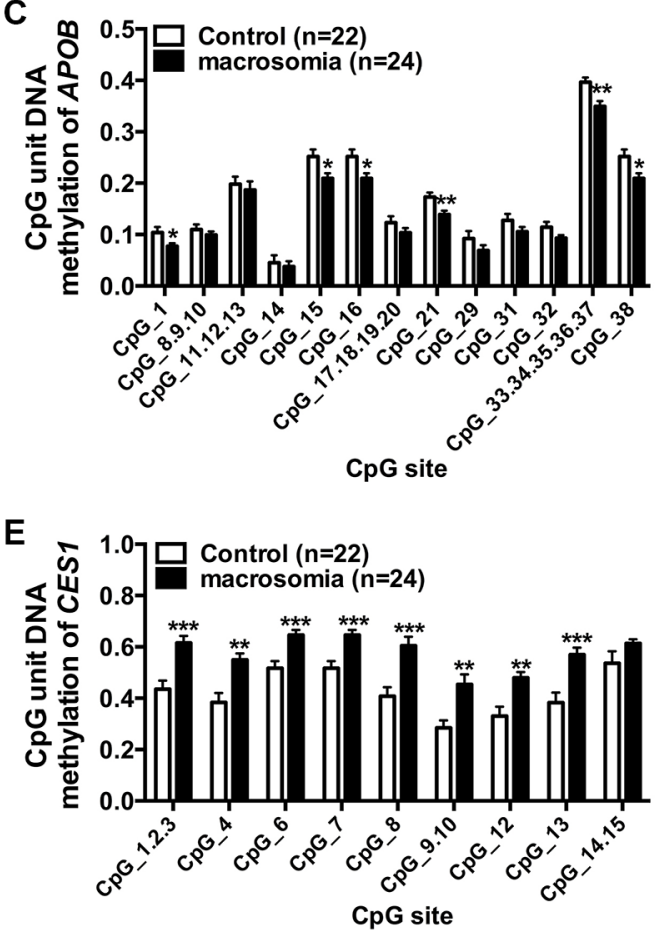

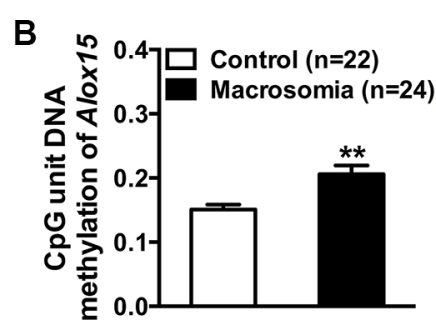

\section{D}

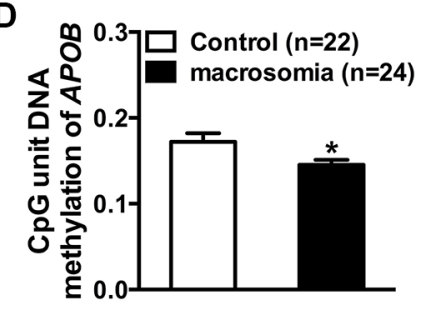

$\mathbf{F}$

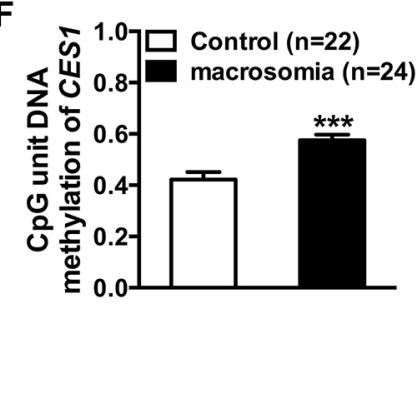

Figure 4: Verification of the target genes. (A and $\mathbf{C}$ and $\mathbf{E}$ ) Percentage of DNA methylation of individual $\mathrm{CpG}$ sites within the island at $A L O X 15, A P O B$, and CES1 (MASSARRAY) in controls $(n=22)$ and macrosomia $(n=24)$. (B and $\mathbf{D}$ and $\mathbf{F})$ Median of $\%$ DNA methylation for each region in controls $(n=22)$ and macrosomia $(n=24)$. Values (in A-F) are expressed as means $\pm \mathrm{SE}, * * * p<0.0001 * * p<0.01$, ${ }^{*} p<0.05$, compared with the corresponding control. 
has not been possible to increase the number of samples analyzed by the $450 \mathrm{~K}$ plat-form in consideration of the cost of such an epigenome-wide study. It resulted in limited statistical power, weakening conclusions on methylation alteration of the individual $\mathrm{CpG}$ sites. Despite these limitations, verifying methylation differences on more $\mathrm{CpG}$ sites nearby enlarged samples and applying systematic functional analysis contributed to increasing our confidence in the results.

High birth weight contributed to epigenetic changes in embryonic organ development and morphogenesis, which probably influence an offspring's future health. Endocytic processes, enriched in KEGG pathway analysis is a responsive mechanism to variable environments in the uterus and after birth. It (including autophagy) was linked to a wide array of vascular processes, ranging from angiogenesis to calcification of the vessel wall. Alterations in autophagic flux are also increasingly being implicated in disease processes, including atherosclerosis and pulmonary hypertension [36].

As suggested by common disease and disorder pathway analyses results, a number of the identified epivariations were correlated with CVD candidate genes. DNA methylation at gene promoter regions is often associated with transcriptional repression due to interactions between DNA methylation, methyl DNA binding proteins, and histone deacetyltransferases. In contrast, promoter hypomethylation is often associated with a euchromatic state and transcriptional permissiveness [37]. Apolipoprotein B $(A P O B)$ transcribes and translates into the main Apo lipoprotein of chylomicrons and LDL. LDL is considered as one of the main molecules leading to atherosclerosis and associated with cardiovascular risk [38]. Higher methylation levels in $A P O B$ were reported to be associated with an increased risk of having a birth weight below the 50th percentile [29]. The same relationship of methylation levels in $A P O B$ and birth weight was found in our study. Lower $A P O B$ methylation levels in specific CpGs located on islands were detected in the macrosomia group by $450 \mathrm{~K}$. We detected methylation of $399 \mathrm{bp}$ DNA surrounding the MVPs in enlarged samples by MASSARRAY and identified the same methylation differences. Delta-like homolog 1 (DLK1), an imprinted gene, is subject to multiple levels of epigenetic dosage control beyond conventional mechanisms of tissueand temporal specific regulation [39]. Upregulation of DLK1 impairs angiogenesis by inhibiting Endothelial cell (EC) proliferation [40] and impedes the regenerative response of ECs to the proapoptotic and antiproliferative effects of oxidized LDL [41]. CES1, carboxylesterase 1 , encodes a member of the carboxylesterase large family and participates in fatty acyl and cholesterol ester metabolism. An in vitro study showed that overexpression of CES1 in THP-1 macrophages markedly increases the rate of cholesterol efflux. Overexpression of human
CES1 in macrophages increases the recruiting rates of macrophage and reduces atherosclerosis in Western dietfed Ldlr-/-mice [42]. Differential methylation in the island and surround of CES1 was quite probably resulted in expression change [43].

In conclusion, our results suggest that high maternal TG level will dysregulate the fetal epigenome and mediate the increase of cardiometabolic disease risk in later life. Neonate born LGA presented high maternal BMI and TG levels during pregnancy. Individuals with high birth weight were accompanied by a specific pattern change of DNA methylation, including CVD candidate genes. Our data therefore provide supportive evidence that DNA methylation is involved in fetal cardiometabolic programming. The candidate genes that we have identified in this study might be severs as potential biomarkers to assess the risk of cardiometabolic disease. Therefore, our findings in this study not only extend our knowledge of pathomechanism of cardiometabolic diseases but also hold great promise for future clinical applications.

\section{MATERIALS AND METHODS}

\section{Study population}

Preschool children who were born at term (gestational age $\geq 37$ and $<42$ weeks) after uncomplicated pregnancies and in the absence of perinatal illness were invited to participate in the study between January and December 2012 from the Child Care Center of Women's Hospital, School of Medicine, Zhejiang University. Exclusion criteria were multiple gestations, congenital anomalies, preterm infants, and small-for-gestational age. Subjects were divided according to birth weight: AGA, between 10th and 90th percentile, and LGA, > 90th percentile [44]. Fifty-eight children aged 3-6 years born LGA were enrolled according the criteria; 123 subjects born AGA were matched approximately at a ratio of $1: 2$ to the LGA group according to maternal age $( \pm 1$ year) and maternal weight gain $( \pm 1 \mathrm{~kg})$ during pregnancy. The study was approved by the Research and Ethics Committee of the Zhejiang Women's Hospital, School of Medicine, Zhejiang University, Hangzhou, China, and was registered in the Chinese Clinical Trial Registry (ChicCTROCH-14004536, www.medresman.org). Informed consent from the parents of each participant was obtained.

The umbilical cord blood of neonates born in the Women's Hospital, School of Medicine, Zhejiang University, was routinely collected and preserved in the biomedical sample center. The corresponding maternal and birth information was investigated. Among the enrolled subjects, 12 umbilical cord blood samples were collected at delivery, randomly involving six normal birth weight (AGA) and six macrosomia newborns, from the sample center for the DNA methylation analysis. 


\section{Serum biochemistry parameters analysis}

Serum samples were obtained under fasting conditions in the early morning and were performed for the concentration metabolic assessment. The assays for serum lipids and peripheral glucose were assayed by the clinical chemistry laboratory at the Women's Hospital, School of Medicine, Zhejiang University (Abbott C1600, Chicago, IL, USA). The fasting insulin was tested at the same time using a chemiluminescence immunoassay (Access 2; Beckman Coulter, Fullerton, CA, USA). The homeostatic model assessment (HOMA) IR was calculated by dividing the product of insulin (microunits per milliliter) and glucose (millimoles per liter) by 22.5 [45].

\section{DNA preparation}

Directly after delivery, umbilical cord blood was collected and stored at $-80^{\circ} \mathrm{C}$. DNA was extracted from buffy coats using the QIAamp DNA Mini Kit (Qiagen, Valencia, CA, USA), according to manufacturer's protocol. Following isolation, all samples were checked for DNA quality and quantity. Those with good quality (260/280 ratio exceeding 1.8) and DNA concentration $\geq 50 \mathrm{ng} / \mu \mathrm{l}$ were considered to be qualified.

\section{K BeadChip DNA methylation analysis}

DNA methylation was measured using the IlluminaInfinium HumanMethylation450 BeadChips (Illumina, San Diego, CA, USA). For each sample, $500 \mathrm{ng}$ of DNA was bisulfite converted using the EZ DNA Methylation Kit (Qiagen) and analyzed on HM450 Bead Chips, both according to the manufacturers' instructions. The Illumina Genome Studio program was used for normalization and extraction of the methylated and unmethylated signal intensities. Briefly, a probe specific to each "allele" (methylated vs. unmethylated cytosines) was designed. Then, a single base extension of the probes incorporated a labeled ddNTP. Each probe signal was then used to compute a $\beta$ value ( $\beta$ ), which was a quantitative measure of DNA methylation ranging from 0 (no cytosine methylation) to 1 (complete cytosine methylation). Concretely, $\beta$ was calculated as: $\beta=$ intensity of the methylated allele (M) / (intensity of the unmethylated allele (U) + intensity of the methylated allele (M) + 100) [46]. Quality controls were conducted according to the manufacturer's recommendations. Steps of DNP and Biotin staining, bisulfite conversion, extension, hybridization, target removal, and negative and nonpolymorphic controls were included.

\section{Chip analysis methylation pipeline (ChAMP)}

Analysis of $450 \mathrm{~K}$ was performed according to the ChAMP package [47]. In brief, after raw data was loaded, three quality control images were provided. First, ChAMP filtered the data for detection $(p<0.01)$. Then, probes with a bead count $<3$ in at least $5 \%$ of samples per probe were filtered out $(n=3324)$. An additional probe on the panel that contained single nucleotide polymorphisms (SNPs) or repetitive elements was removed to avoid their interference effect on measurement of DNA methylation $(n=37730)$. To adjust for type- 2 bias, data were normalized with Beta-mixture quantile normalization (BMIQ). Meanwhile, an adjusted $p$-value was calculated for differential methylation using a linear model. Methylation-variable positions (MVPs) were identified for appropriate contrasts and target genes, for which MVP mapping served for bioinformatics analysis.

\section{DNA methylation validation}

$\begin{array}{clcc}\begin{array}{c}\text { Differential methylation of Cytosine- } \\ \text { phosphate-guanines }\end{array} & \text { (CpG) was validated using }\end{array}$ MassARRAYEpiTYPER assays (Sequenom, San Diego, CA, USA). We designed three primer sets by EpiDesigner software (http://epidesigner.com) to cover MVPs of gene $A L O X 15, A P O B$, and CES1 (Supplementary Table S6). In total, 6 CpGs of $A L O X 15,38 \mathrm{CpGs}$ of $A P O B$, and $13 \mathrm{CpGs}$ of $C E S 1$ were included in the target products, respectively. Each reverse primer was designed to contain a $\mathrm{T} 7$ promoter tag for in vitro transcription, and each forward primer incorporated a 10-mer tag to adjust for melting temperature differences. According to the manufacturer's standard protocol (Sequenom), bisulfiteconverted genomic DNA was prepared for polymerase chain reaction (PCR). Amplification parameters were set as follows: $95^{\circ} \mathrm{C}$ for $5 \mathrm{~min}, 94^{\circ} \mathrm{C}$ for $20 \mathrm{sec}, 60^{\circ} \mathrm{C}$ for $25 \mathrm{sec}$, and $72^{\circ} \mathrm{C}$ for $1 \mathrm{~min}$ for a total of 40 cycles, with a final incubation at $72^{\circ} \mathrm{C}$ for $5 \mathrm{~min}$. PCR products were used in in vitro transcription reactions (T-cleavage assay). Samples were then spotted on a 384-SpectroCHIP (Sequenom) followed by spectral acquisition on a MassARRAY analyzer compact MALDI-TOF-MS (Sequenom). Methylation data of individual units (one to three $\mathrm{CpG}$ sites per unit) was generated by the Epitope software (Sequenom). The non-applicable reading and its corresponding site were eliminated in calculations.

\section{Statistical analyses}

The clinical data were normally distributed and expressed as mean \pm standard deviation (SD). The independent-samples $t$-test, non-parametric test, and chi-square tests were used to evaluate the statistical significance between the two groups. Associations between parameters were assessed using the Pearson correlation coefficient. Partial correlation was used to control confounding variables. Multiple linear regression analysis, using BP values and metabolic parameters as dependent variables and birth weight, current weight, average weight 
gain as independent variables, was calculated. Statistical analyses were performed using SPSS version 19.0 for Windows. $P$ values $<0.05$ were considered statistically significant with a statistical power of $80 \%$.

\section{ACKNOWLEDGMENTS AND FUNDING}

We would like to thank our patients as well as the doctors and nurses who had assisted with recruitment for this study. This study was sponsored by grants from the National Basic Research Program of China (No.2012CB944900 and 31471405 and 81450038 to H.F.H., No. 81501340 to X.H.L, No.81270708 to J.Z.S, No.81401219 to J.Y.Z, No.81601238 to L.G, and NO.81501276 to Y.Q.X). Shanghai Hospital Development Center (No. SHDC12014131 to H.F.H.). Shanghai Municipal Commission of Health and family planning (No.20164Y0251 to L.G and NO. 20154Y0039 to Y.Q.X),

\section{CONFLICTS OF INTEREST}

The authors declare they have no conflicts of interest.

\section{REFERENCES}

1. Yu Z, Han S, Zhu J, Sun X, Ji C, Guo X. Pre-pregnancy body mass index in relation to infant birth weight and offspring overweight/obesity: a systematic review and meta-analysis. PLoS One. 2013; 8:e61627.

2. Baird J, Fisher D, Lucas P, Kleijnen J, Roberts H, Law C. Being big or growing fast: systematic review of size and growth in infancy and later obesity. BMJ. 2005; 331:929.

3. Fujita Y, Kouda K, Nakamura H, Iki M. Association of rapid weight gain during early childhood with cardiovascular risk factors in Japanese adolescents. J Epidemiol. 2013; 23:103-108.

4. Evagelidou EN, Giapros VI, Challa AS, Cholevas VK, Vartholomatos GA, Siomou EC, Kolaitis NI, Bairaktari ET, Andronikou SK. Prothrombotic state, cardiovascular, and metabolic syndrome risk factors in prepubertal children born large for gestational age. Diabetes Care. 2010; 33:2468-2470.

5. Dabelea D, Hanson RL, Lindsay RS, Pettitt DJ, Imperatore G, Gabir MM, Roumain J, Bennett PH, Knowler WC. Intrauterine exposure to diabetes conveys risks for type 2 diabetes and obesity: a study of discordant sibships. Diabetes. 2000; 49:2208-2211.

6. Boney CM, Verma A, Tucker R, Vohr BR. Metabolic syndrome in childhood: association with birth weight, maternal obesity, and gestational diabetes mellitus. Pediatrics. 2005; 115:e290-296.

7. Eriksson JG, Forsen T, Tuomilehto J, Osmond C, Barker DJ. Early growth and coronary heart disease in later life: longitudinal study. BMJ. 2001; 322:949-953.
8. Bhargava SK, Sachdev HS, Fall CH, Osmond C, Lakshmy R, Barker DJ, Biswas SK, Ramji S, Prabhakaran D, Reddy KS. Relation of serial changes in childhood body-mass index to impaired glucose tolerance in young adulthood. N Engl J Med. 2004; 350:865-875.

9. Law CM, Shiell AW, Newsome CA, Syddall HE, Shinebourne EA, Fayers PM, Martyn CN and de Swiet M. Fetal, infant, and childhood growth and adult blood pressure: a longitudinal study from birth to 22 years of age. Circulation. 2002; 105:1088-1092.

10. Lurbe E, Garcia-Vicent C, Torro MI, Aguilar F, Redon J. Associations of birth weight and postnatal weight gain with cardiometabolic risk parameters at 5 years of age. Hypertension. 2014; 63:1326-1332.

11. Youngson NA, Whitelaw E. Transgenerational epigenetic effects. Annu Rev Genomics Hum Genet. 2008; 9:233-257.

12. Heijmans BT, Tobi EW, Stein AD, Putter H, Blauw GJ, Susser ES, Slagboom PE, Lumey LH. Persistent epigenetic differences associated with prenatal exposure to famine in humans. Proc Natl Acad Sci U S A. 2008; 105:1704617049.

13. Tobi EW, Lumey LH, Talens RP, Kremer D, Putter H, Stein AD, Slagboom PE, Heijmans BT. DNA methylation differences after exposure to prenatal famine are common and timing- and sex-specific. Hum Mol Genet. 2009; 18:4046-4053.

14. Godfrey KM, Sheppard A, Gluckman PD, Lillycrop KA, Burdge GC, McLean C, Rodford J, Slater-Jefferies JL, Garratt E, Crozier SR, Emerald BS, Gale CR, Inskip HM, et al. Epigenetic gene promoter methylation at birth is associated with child's later adiposity. Diabetes. 2011; 60:1528-1534.

15. Jaenisch R, Bird A. Epigenetic regulation of gene expression: how the genome integrates intrinsic and environmental signals. Nat Genet. 2003; 33:245-254.

16. Wong CC, Caspi A, Williams B, Craig IW, Houts R, Ambler A, Moffitt TE, Mill J. A longitudinal study of epigenetic variation in twins. Epigenetics. 2010; 5:516-526.

17. Falaschetti E, Hingorani AD, Jones A, Charakida M, Finer N, Whincup P, Lawlor DA, Davey Smith G, Sattar N, Deanfield JE. Adiposity and cardiovascular risk factors in a large contemporary population of pre-pubertal children. Eur Heart J. 2010; 31:3063-3072.

18. Lawlor DA, Benfield L, Logue J, Tilling K, Howe LD, Fraser A, Cherry L, Watt P, Ness AR, Davey Smith G, Sattar N. Association between general and central adiposity in childhood, and change in these, with cardiovascular risk factors in adolescence: prospective cohort study. BMJ. 2010; 341:c6224.

19. Corvalan C, Uauy R, Stein AD, Kain J, Martorell R. Effect of growth on cardiometabolic status at $4 \mathrm{y}$ of age. The Am J Clin Nutr. 2009; 90:547-555.

20. Barker DJ, Osmond C, Law CM. The intrauterine and early postnatal origins of cardiovascular disease and chronic 
bronchitis. J Epidemiol Community Health. 1989; 43: 237-240.

21. Barker DJ. Fetal origins of coronary heart disease. BMJ. 1995; 311:171-174.

22. Barker DJ. The developmental origins of well-being. Philos Trans R Soc Lond B Biol Sci. 2004; 359:1359-1366.

23. Barker DJ, Winter PD, Osmond C, Margetts B, Simmonds SJ. Weight in infancy and death from ischaemic heart disease. Lancet. 1989; 2:577-580.

24. Paneth N, Susser M. Early origin of coronary heart disease (the "Barker hypothesis"). BMJ. 1995; 310:411-412.

25. Palatianou ME, Simos YV, Andronikou SK, Kiortsis DN. Long-term metabolic effects of high birth weight: a critical review of the literature. Horm Metab Res. 2014; 46:911920.

26. Zhang Y, Li H, Liu SJ, Fu GJ, Zhao Y, Xie YJ, Zhang Y, Wang YX. The associations of high birth weight with blood pressure and hypertension in later life: a systematic review and meta-analysis. Hypertens Res. 2013; 36:725-735.

27. Mamun AA, O'Callaghan M, Callaway L, Williams G, Najman J, Lawlor DA. Associations of gestational weight gain with offspring body mass index and blood pressure at 21 years of age: evidence from a birth cohort study. Circulation. 2009; 119:1720-1727.

28. Huang HF, Sheng JZ. Gamete and Embryo-fetal Origins of Adult Diseases. Springer, New York. 2014.

29. Haworth KE, Farrell WE, Emes RD, Ismail KM, Carroll WD, Borthwick HA, Yates AM, Hubball E, Rooney A, Khanam M, Aggarwal N, Jones PW, Fryer AA. Combined influence of gene-specific cord blood methylation and maternal smoking habit on birth weight. Epigenomics. 2013; 5:37-49.

30. Dominguez-Salas P, Cox SE, Prentice AM, Hennig BJ, Moore SE. Maternal nutritional status, C(1) metabolism and offspring DNA methylation: a review of current evidence in human subjects. Proc Nutr Soc. 2012; 71:154-165.

31. Ding GL, Wang FF, Shu J, Tian S, Jiang Y, Zhang D, Wang N, Luo Q, Zhang Y, Jin F, Leung PC, Sheng JZ, Huang HF. Transgenerational glucose intolerance with Igf2/H19 epigenetic alterations in mouse islet induced by intrauterine hyperglycemia. Diabetes. 2012; 61:1133-1142.

32. Jirtle RL, Skinner MK. Environmental epigenomics and disease susceptibility. Nat Rev Genet. 2007; 8:253-262.

33. Ruchat SM, Houde AA, Voisin G, St-Pierre J, Perron P, Baillargeon JP, Gaudet D, Hivert MF, Brisson D, Bouchard L. Gestational diabetes mellitus epigenetically affects genes predominantly involved in metabolic diseases. Epigenetics. 2013; 8:935-943.

34. Teschendorff AE, Marabita F, Lechner M, Bartlett T, Tegner J, Gomez-Cabrero D, Beck S. A beta-mixture quantile normalization method for correcting probe design bias in Illumina Infinium $450 \mathrm{k}$ DNA methylation data. Bioinformatics. 2013; 29:189-196.
35. Marabita F, Almgren M, Lindholm ME, Ruhrmann S, Fagerstrom-Billai F, Jagodic M, Sundberg CJ, Ekstrom TJ, Teschendorff AE, Tegner J, Gomez-Cabrero D. An evaluation of analysis pipelines for DNA methylation profiling using the Illumina HumanMethylation450 BeadChip platform. Epigenetics. 2013; 8:333-346.

36. Nussenzweig SC, Verma S, Finkel T. The role of autophagy in vascular biology. Circ Res. 2015; 116:480-488.

37. Murphy SK, Yang H, Moylan CA, Pang H, Dellinger A, Abdelmalek MF, Garrett ME, Ashley-Koch A, Suzuki A, Tillmann HL, Hauser MA, Diehl AM. Relationship between methylome and transcriptome in patients with nonalcoholic fatty liver disease. Gastroenterology. 2013; 145:1076-1087.

38. Thomas GS, Cromwell WC, Ali S, Chin W, Flaim JD, Davidson M. Mipomersen, an apolipoprotein B synthesis inhibitor, reduces atherogenic lipoproteins in patients with severe hypercholesterolemia at high cardiovascular risk: a randomized, double-blind, placebo-controlled trial. J Am Coll Cardiol. 2013; 62:2178-2184.

39. Takada S, Tevendale M, Baker J, Georgiades P, Campbell E, Freeman T, Johnson MH, Paulsen M, Ferguson-Smith AC. Delta-like and gtl2 are reciprocally expressed, differentially methylated linked imprinted genes on mouse chromosome 12. Curr Biol. 2000; 10:1135-1138.

40. Wang S, Aurora AB, Johnson BA, Qi X, McAnally J, Hill JA, Richardson JA, Bassel-Duby R, Olson EN. The endothelial-specific microRNA miR-126 governs vascular integrity and angiogenesis. Dev Cell. 2008; 15:261-271.

41. Colles SM, Maxson JM, Carlson SG, Chisolm GM. Oxidized LDL-induced injury and apoptosis in atherosclerosis. Potential roles for oxysterols. Trends Cardiovasc Med. 2001; 11:131-138.

42. Zhao B, Song J, St Clair RW, Ghosh S. Stable overexpression of human macrophage cholesteryl ester hydrolase results in enhanced free cholesterol efflux from human THP1 macrophages. Am J Physiol Cell Physiol. 2007; 292:C405-412.

43. Hori T, Hosokawa M. DNA methylation and its involvement in carboxylesterase 1A1 (CES1A1) gene expression. Xenobiotica. 2010; 40:119-128.

44. Battaglia FC, Lubchenco LO. A practical classification of newborn infants by weight and gestational age. J Pediatr. 1967; 71:159-163.

45. Haffner SM, Miettinen H, Stern MP. The homeostasis model in the San Antonio Heart Study. Diabetes Care. 1997; 20:1087-1092.

46. Bibikova M, Barnes B, Tsan C, Ho V, Klotzle B, Le JM, Delano D, Zhang L, Schroth GP, Gunderson KL, Fan JB, Shen R. High density DNA methylation array with single CpG site resolution. Genomics. 2011; 98:288-295.

47. Morris TJ, Butcher LM, Feber A, Teschendorff AE, Chakravarthy AR, Wojdacz TK, Beck S. ChAMP: 450k Chip Analysis Methylation Pipeline. Bioinformatics. 2014; 30:428-430. 